\title{
Research on Improved Control Strategy for STATCOM Based on Virtual Matrix Method
}

\author{
Xudong Wang ${ }^{1, a}$, Shengtie Wang ${ }^{1}$ \\ ${ }^{1}$ College of Electric Power, Inner Mongolia University of Technology, 010080 Hohhot, China
}

\begin{abstract}
Fast and accurate detection of reactive current is the precondition for the realization of static synchronous compensator (STATCOM) reactive power compensation and harmonic suppression. Aiming at deviation and delay of the traditional reactive current detection algorithm with phase-locked loop (PLL) and low-pass filter (LPF) of STATCOM, a novel improved reactive current detection algorithm without PLL is proposed, in which the virtual matrix (VM) is built to replace the original PLL, and improved current average value filter is used to realize the function of LPF, so as to improve the real-time performance and robustness of reactive current detection. The realization process of VM detection method is derived in this paper, and improved control strategy for STATCOM is designed based on the VM detection method. Simulation analysis of the proposed detection algorithm and control strategy is conducted in Matlab platform so as to verify the correctness and effectiveness of the control strategy. The VM detection has the advantages of simple structure, fast response and easy for digital realization, which provides reference for the improvement of reactive power compensation precision for STATCOM.
\end{abstract}

\section{Introduction}

With the increase of wind power capacity and the development of micro-grid technology, the structure of power system is becoming more and more complex, which brings higher requirements for power quality of power network [1-4]. Reactive power compensation device is one of the effective ways to solve the problem of power quality. STATCOM is an important part of flexible AC transmission system[5], which is a hot spot in the field of reactive power compensation[6-8].

STATCOM control system is composed of reactive current detection module, control algorithm, pulse trigger module and so on. Fast and accurate detection of reactive current is the premise of realizing STATCOM reactive power compensation and harmonic suppression [9-11]. The $p-q$ and $i_{p}-i_{q}$ detection method based on instantaneous reactive power theory has the effect of voltage distortion and impact load, and the response speed and robustness of the system are reduced[12-14]. In [15], a software PLL is designed to replace the traditional PLL, but the error caused by the phase change of the grid voltage is not considered in this paper. In [16], the concept of "intelligent impedance" is introduced, and the function of the PLL is replaced by the intelligent impedance and proportional resonant controller. However, this method brings higher requirements for the design of parameters, which is not easy to achieve. In [17], synchronous rotation angular velocity is preset, so as to find the

\footnotetext{
${ }^{a}$ Corresponding author :wxd010353@imut.edu.cn 
way to replace the PLL by sine/cosine function table lookup, but the error caused by the grid voltage phase change is not eliminated. In [18-19], the neural network controller is designed to replace the traditional PLL, but the response speed and the number of iterations are important factors that affect the performance of the algorithm. In [20], the fundamental positive sequence voltage extraction module is used for detecting the fundamental positive sequence voltage to replace the original PLL, but the introduction of two low pass filter reduces the real-time performance of the detection.

A new method for improving the reactive current detection based on VM is proposed in this paper. In this algorithm, the VM is used to replace the original PLL, and the current average value method is adopted to replace the LPF. The improved control strategy for STATCOM is designed based on VM reactive current detection algorithm. The effectiveness of the proposed algorithm and control strategy is verified by Matlab simulation.

\section{Virtual Matrix Method}

Assuming three-phase detection currents are:

$$
\left[\begin{array}{c}
i_{\mathrm{a}} \\
i_{\mathrm{b}} \\
i_{\mathrm{c}}
\end{array}\right]=\sqrt{2}\left[\begin{array}{c}
\sum_{m=1}^{\infty} I_{m} \sin \left(m \omega t+\theta_{m}\right) \\
\sum_{m=1}^{\infty} I_{m} \sin \left(m \omega t-\frac{2 m \pi}{3}+\theta_{m}\right) \\
\sum_{m=1}^{\infty} I_{m} \sin \left(m \omega t+\frac{2 m \pi}{3}+\theta_{m}\right)
\end{array}\right]
$$

$i_{\mathrm{a}}, i_{\mathrm{b}}$ and $i_{\mathrm{c}}$ are three-phase detected currents; $I_{\mathrm{m}}$ and $\theta_{\mathrm{m}}$ are effective values of each measured harmonic current and the initial phase angle; $m$ is the number of harmonics.

According to the traditional $\mathrm{i}_{\mathrm{p}}-\mathrm{i}_{\mathrm{q}}$ detection method:

$$
\left[\begin{array}{c}
i_{\mathrm{p}} \\
i_{\mathrm{q}}
\end{array}\right]=\mathbf{C C}_{32}\left[\begin{array}{c}
i_{\mathrm{a}} \\
i_{\mathrm{b}} \\
i_{\mathrm{c}}
\end{array}\right]=\sqrt{\frac{2}{3}} \mathbf{C}\left[\begin{array}{ccc}
1 & -1 / 2 & -1 / 2 \\
0 & \sqrt{3} / 2 & -\sqrt{3} / 2
\end{array}\right]\left[\begin{array}{c}
i_{\mathrm{a}} \\
i_{\mathrm{b}} \\
i_{\mathrm{c}}
\end{array}\right]
$$

C is the coefficient matrix obtained by the PLL circuit.

In order to simplify the detection circuit, the virtual matrix $\mathbf{C}_{\mathbf{v}}$ is introduced to replace matrix $\mathbf{C}$. The expression of $\mathbf{C}_{\mathbf{v}}$ is:

$$
\mathbf{C}_{v}=\left[\begin{array}{cc}
\sin (\omega t+\theta) & -\cos (\omega t+\theta) \\
-\cos (\omega t+\theta) & -\sin (\omega t+\theta)
\end{array}\right]
$$

$\theta$ is an arbitrary initial phase angle.

The matrix $\mathbf{C}_{\mathbf{v}}$ is substituted into the expression (2), then:

$$
\left[\begin{array}{l}
i_{\mathrm{p}} \\
i_{\mathrm{q}}
\end{array}\right]=\mathbf{C}_{v} \mathbf{C}_{32}\left[\begin{array}{l}
i_{\mathrm{a}} \\
i_{\mathrm{b}} \\
i_{\mathrm{c}}
\end{array}\right]=\sqrt{3}\left[\begin{array}{l}
\sum_{n=1}^{\infty} I_{n} \cos \left[(n \pm 1) \omega t+\theta_{n}-\theta\right] \\
-\sum_{n=1}^{\infty} I_{n} \sin \left[(n \pm 1) \omega t+\theta_{n}-\theta\right]
\end{array}\right]
$$

When $n=1$, the DC components of $i_{\mathrm{p}}$ and $i_{\mathrm{q}}$ are: 


$$
\left[\begin{array}{c}
i_{\mathrm{pm}} \\
\mathrm{i}_{\mathrm{qm}}
\end{array}\right]=\sqrt{3}\left[\begin{array}{c}
I_{1} \cos \left(\theta_{1}-\theta\right) \\
-I_{1} \sin \left(\theta_{1}-\theta\right)
\end{array}\right]
$$

Three-phase fundamental currents are:

$$
\begin{gathered}
{\left[\begin{array}{c}
i_{\mathrm{af}} \\
i_{\mathrm{bf}} \\
i_{\mathrm{cf}}
\end{array}\right]=\mathbf{C}_{23} \mathbf{C}_{v}^{-1}\left[\begin{array}{c}
i_{\mathrm{pm}} \\
i_{\mathrm{qm}}
\end{array}\right]=\sqrt{2}\left[\begin{array}{c}
I_{1} \sin \left(\omega t+\theta_{1}\right) \\
I_{1} \sin \left(\omega t-\frac{2 \pi}{3}+\theta_{1}\right) \\
I_{1} \sin \left(\omega t+\frac{2 \pi}{3}+\theta_{1}\right)
\end{array}\right]} \\
{\left[\begin{array}{c}
i_{\mathrm{afp}} \\
i_{\mathrm{bfp}} \\
i_{\mathrm{cfp}}
\end{array}\right]=\sqrt{2}\left[\begin{array}{c}
I_{1} \sin (\omega t+\theta) \\
I_{1} \sin \left(\omega t-\frac{2 \pi}{3}+\theta\right) \\
I_{1} \sin \left(\omega t+\frac{2 \pi}{3}+\theta\right)
\end{array}\right] \cos \left(\theta_{1}-\theta\right)} \\
{\left[\begin{array}{c}
i_{\mathrm{afq}} \\
i_{\mathrm{bfq}} \\
i_{\mathrm{cfq}}
\end{array}\right]=\sqrt{2}\left[\begin{array}{c}
I_{1} \cos (\omega t+\theta) \\
I_{1} \cos \left(\omega t-\frac{2 \pi}{3}+\theta\right) \\
I_{1} \cos \left(\omega t+\frac{2 \pi}{3}+\theta\right)
\end{array}\right] \sin \left(\theta_{1}-\theta\right)}
\end{gathered}
$$

$i_{\mathrm{afp}}, i_{\mathrm{bfp}}$ and $i_{\mathrm{cfp}}$ are active components of three-phase fundamental currents; $i_{\mathrm{afq}}, i_{\mathrm{bfq}}$ and $i_{\mathrm{cfq}}$ are reactive components of three-phase fundamental currents.

According to the expression (6)-(7), it is still capable of detecting the fundamental active and reactive components of the detected current without errors. Compensation instruction current can be expressed as follows:

$$
\left[\begin{array}{l}
i_{\text {aref }} \\
i_{\text {bref }} \\
i_{\text {cref }}
\end{array}\right]=\left[\begin{array}{l}
i_{\mathrm{a}} \\
i_{\mathrm{b}} \\
i_{\mathrm{c}}
\end{array}\right]-\left[\begin{array}{l}
i_{\mathrm{afp}} \\
i_{\mathrm{bfp}} \\
i_{\text {cfp }}
\end{array}\right]
$$

Similarly, assuming the three-phase supply voltage are:

$$
\left[\begin{array}{l}
e_{\mathrm{a}} \\
e_{\mathrm{b}} \\
e_{\mathrm{c}}
\end{array}\right]=\sqrt{2}\left[\begin{array}{c}
\sum_{n=1}^{\infty} E_{1 n} \sin (n \omega t)+\sum_{n=1}^{\infty} E_{2 n} \sin \left(n \omega t+\theta_{2 n}\right) \\
\sum_{n=1}^{\infty} E_{1 n} \sin \left(n \omega t-\frac{2 \pi}{3}\right)+\sum_{n=1}^{\infty} E_{2 n} \sin \left(n \omega t+\frac{2 \pi}{3}+\theta_{2 n}\right) \\
\sum_{n=1}^{\infty} E_{1 n} \sin \left(n \omega t+\frac{2 \pi}{3}\right)+\sum_{n=1}^{\infty} E_{2 n} \sin \left(n \omega t-\frac{2 \pi}{3}+\theta_{2 n}\right)
\end{array}\right]
$$

$e_{\mathrm{a}}, e_{\mathrm{b}}$ and $e_{\mathrm{c}}$ are three-phase voltages; $E_{1 \mathrm{n}}$ and $E_{2 \mathrm{n}}$ are the effective values of positive and negative sequence components of supply voltage harmonics. 
According to the traditional $\mathrm{i}_{\mathrm{p}}-\mathrm{i}_{\mathrm{q}}$ detection method:

$$
\left[\begin{array}{l}
e_{\mathrm{p}} \\
e_{\mathrm{q}}
\end{array}\right]=\sqrt{3}\left[\begin{array}{l}
\sum_{n=1}^{\infty} E_{1 n} \cos [(n-1) \omega t-\theta]+\sum_{n=1}^{\infty} E_{2 n} \cos \left[(n+1) \omega t+\theta_{2 n}+\theta\right] \\
-\sum_{n=1}^{\infty} E_{1 n} \sin [(n-1) \omega t-\theta]-\sum_{n=1}^{\infty} E_{2 n} \sin \left[(n+1) \omega t+\theta_{2 n}+\theta\right]
\end{array}\right]
$$

When $n=1$, the DC components of $e_{\mathrm{p}}$ and $e_{\mathrm{q}}$ are:

$$
\left[\begin{array}{l}
e_{\mathrm{pm}} \\
\mathrm{e}_{\mathrm{qm}}
\end{array}\right]=\sqrt{3}\left[\begin{array}{c}
E_{1} \cos \theta \\
E_{1} \sin \theta
\end{array}\right]
$$

Fundamental positive sequence voltages are:

$$
\left[\begin{array}{c}
e_{\mathrm{af}} \\
e_{\mathrm{bf}} \\
e_{\mathrm{cf}}
\end{array}\right]=\mathbf{C}_{23} \mathbf{C}_{v}^{-1}\left[\begin{array}{l}
e_{\mathrm{pm}} \\
e_{\mathrm{qm}}
\end{array}\right]=\sqrt{2}\left[\begin{array}{c}
E_{1} \sin (\omega t) \\
E_{1} \sin \left(\omega t-\frac{2 \pi}{3}\right) \\
E_{1} \sin \left(\omega t+\frac{2 \pi}{3}\right)
\end{array}\right]
$$

According to the expression (12), it is still capable of detecting the fundamental active and reactive components of the detected power supply voltage without errors.

Three-phase instantaneous active power is:

$$
p=\left[\begin{array}{c}
e_{\mathrm{pm}} \\
e_{\mathrm{qm}}
\end{array}\right]^{T}\left[\begin{array}{l}
i_{\mathrm{pm}} \\
i_{\mathrm{qm}}
\end{array}\right]=e_{\mathrm{pm}} i_{\mathrm{pm}}+e_{\mathrm{qm}} i_{\mathrm{qm}}
$$

Active components of three-phase fundamental currents are:

$$
\left[\begin{array}{c}
i_{\mathrm{afp}} \\
i_{\mathrm{bfp}} \\
i_{\mathrm{cfp}}
\end{array}\right]=\frac{p}{e_{\mathrm{pm}}^{2}+e_{\mathrm{qm}}^{2}} \mathbf{C}_{23} \mathbf{C}_{v}^{-1}\left[\begin{array}{c}
e_{\mathrm{pm}} \\
e_{\mathrm{qm}}
\end{array}\right]
$$

The simplified schematic diagram of the virtual matrix detection method is shown in Fig. 1.

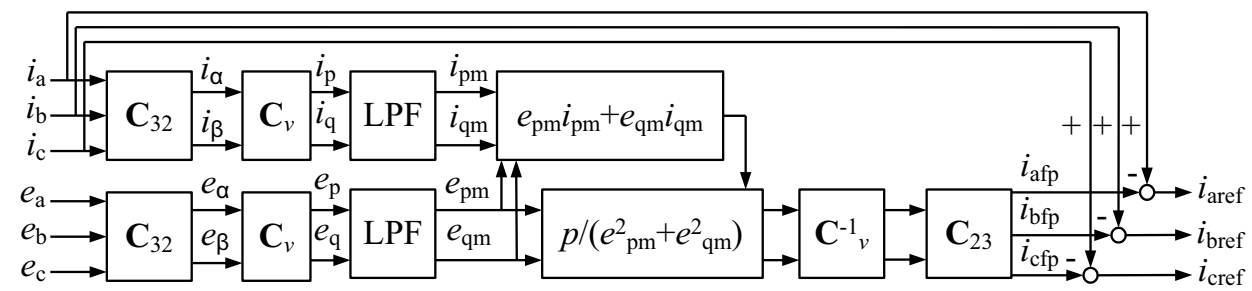

Figure 1. Schematic diagram of VM detection method. 
As shown in Fig. 1, the LPF is used to filter out the AC component of the detected current or voltage signal, and then, the fundamental positive sequence active current or voltage is obtained. Commonly used filters are FIR (Finite Impulse Response) and IIR (Infinite Impulse Response). But both of them have the problems such as complex parameter design, extension of time and so on. In [21], The integral period of traditional current average value method is changed from $\mathrm{T} / 3$ to the least common multiple of various order AC components, so as to filter out all AC components.

According to the expression (4) and (10), if a current or voltage signal does not contain even harmonics, the positive and negative sequence harmonic are $6 k$ and $6 k+6(k=0,1,2, \ldots)$, the minimum common multiple is 6 . The integral period of the current average value filtering method is $T / 6$, that is, the DC component can be obtained by the 1/6 integral cycle time delay. The filter structure is simple and easy to be realized. The schematic diagram of the current average value filter is shown in Fig. 2 .

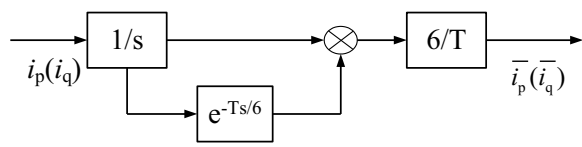

Figure 2. Schematic diagram of current average value filter.

\section{Performance Comparison of Detection Method}

In order to compare the advantages and disadvantages of the proposed method with the traditional $\mathrm{p}-\mathrm{q}$ detection method and $\mathrm{i}_{\mathrm{p}}-\mathrm{i}_{\mathrm{q}}$ detection method, the simulation models of three detection methods are built in Matlab/Simulink platform. In the simulation, it is assumed that the effective value of the power supply voltage is $311 \mathrm{~V}$, the effective value of the fifth and the seventh harmonic are respectively $4 \%$ and $3 \%$ of the effective value of the fundamental wave. The fundamental active value of the measured current is $20 \mathrm{~A}$, while the effective value of the fifth and the seventh harmonic are respectively $4 \%$ and $3 \%$ of the effective value of the fundamental wave. Simulation results are shown in Fig. 3.

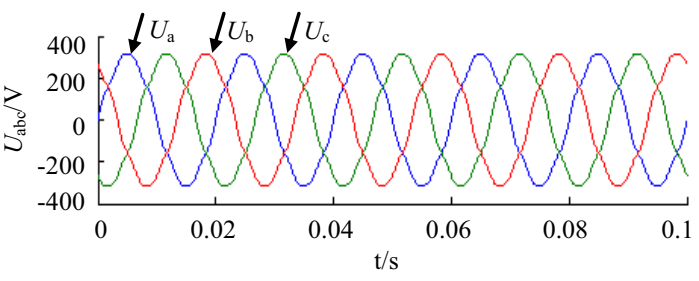

(a) Power supply voltage

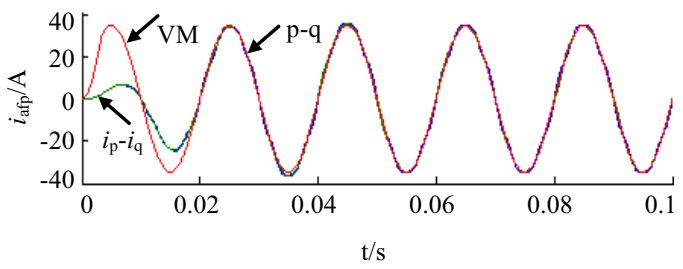

(c) Fundamental active current of A-phase

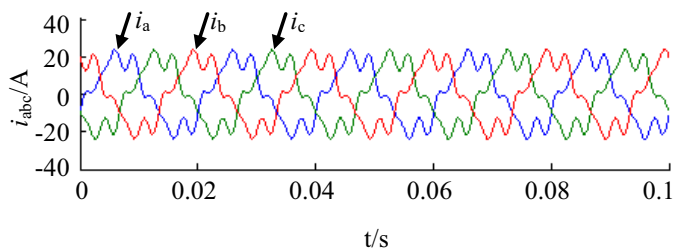

(b) Measured current

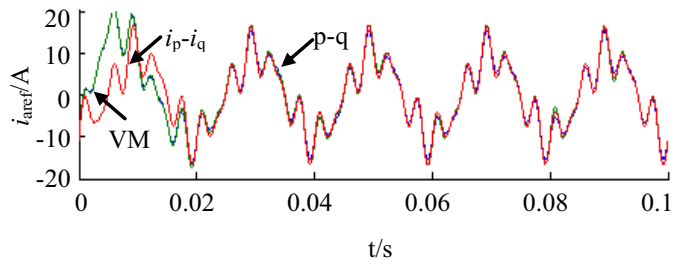

(d) Reactive power and harmonic current of A-phase

Figure 3. Simulation waveforms of three detection methods.

According to the results: 1) There is a certain degree of distortion in the power supply voltage and the measured current, and they contain fifth and seventh harmonic component respectively; 2) when the power supply voltage is distorted, the active current component can not be extracted accurately by p-q method, and the active component of the current can be extracted correctly by $V M$ and $i_{p}-i_{q}$ method; 3 ) both $\mathrm{p}-\mathrm{q}$ and $\mathrm{i}_{\mathrm{p}}-\mathrm{i}_{\mathrm{q}}$ methods require a power cycle to complete the correct acquisition of 
active current components, but the VM method based on current average value filter can achieve the above functions only in $1 / 6$ power cycle; 4) the accuracy and speed of the VM method for reactive power and harmonic current extraction is better than $\mathrm{p}-\mathrm{q}$ method and $\mathrm{i}_{\mathrm{p}} \mathrm{i}_{\mathrm{q}}$ method.

Fast Fourier Transformation (FFT) analysis is performed respectively on the current active component of the three detection methods in Fig. 3. The results are shown in Table 1.

Table 1. The results of FFT analysis.

\begin{tabular}{|c|c|c|}
\hline Method & Effective value of fundamental current $/ \mathbf{A}$ & $\mathbf{T H D} /(\mathbf{\%})$ \\
\hline $\mathrm{p}-\mathrm{q}$ & 18.89 & 5.26 \\
\hline $\mathrm{i}_{\mathrm{p}}-\mathrm{i}_{\mathrm{q}}$ & 18.73 & 2.15 \\
\hline $\mathrm{VM}$ & 19.92 & 0.4 \\
\hline
\end{tabular}

According to the results in Table 1, the fundamental current effective value error of the VM method is $0.4 \%$, while the errors of the other two methods are $5.6 \%$ and $6.35 \%$ respectively. Therefore, the total harmonic distortion rate of the reactive current obtained by the VM method is the smallest, the detection value is the most accurate, and the detection effect is better than the other two detection methods.

\section{Simulation research on STATCOM improved control strategy}

\subsection{Improved control strategy}

According to the schematic diagram of the instruction current detection based on the VM detection method, the improved control strategy for STATCOM is shown in Fig. 4.

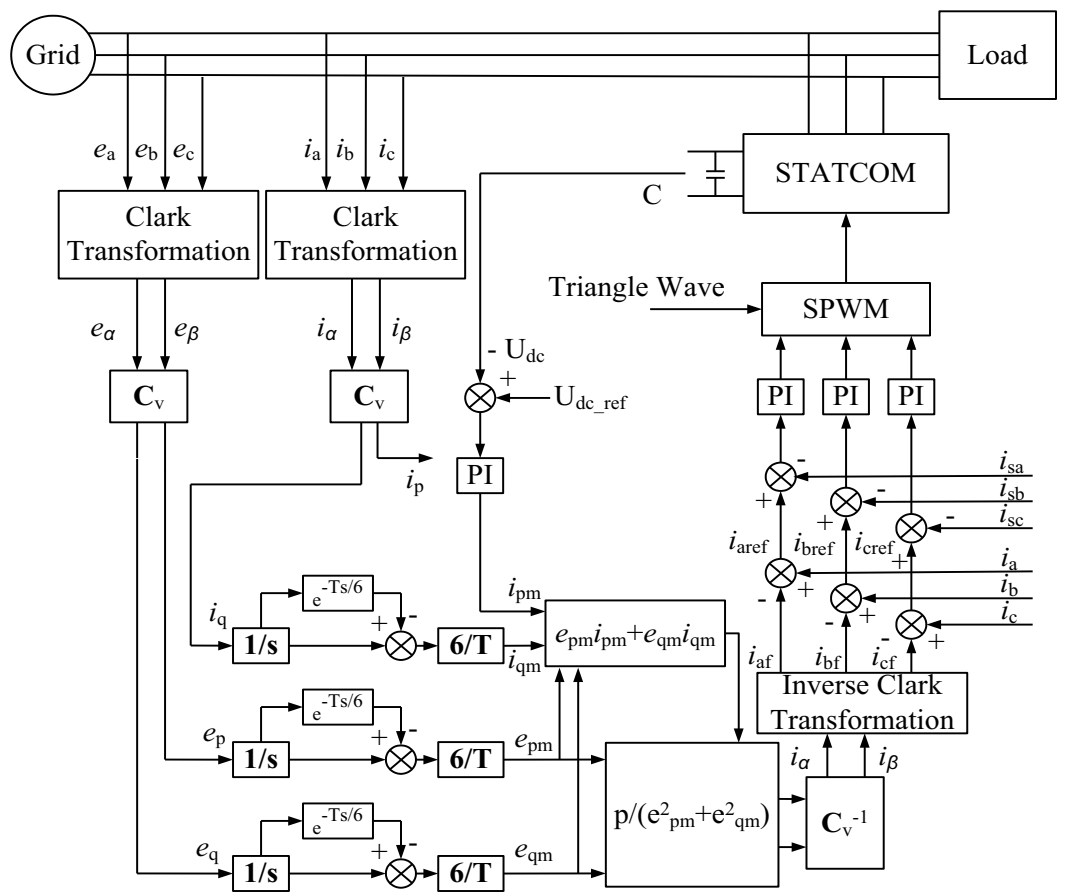

Figure 4. Block diagram of improved control strategy for STATCOM. 
The control strategy is based on double closed-loop decoupling control of voltage outer loop and current inner loop. The instantaneous values of three-phase voltage in the grid side are $e_{\mathrm{a}}, e_{\mathrm{b}}$ and $e_{\mathrm{c}}$ respectively, the instantaneous value of three-phase current are $i_{\mathrm{a}}, i_{\mathrm{b}}$ and $i_{\mathrm{c}}$ respectively. The voltage and current are transformed to the two-phase orthogonal coordinate system, the instantaneous voltage $e_{\alpha}, e_{\beta}$ and the instantaneous current $i_{\alpha}, i_{\beta}$ are obtained respectively. After the $\mathbf{C}_{\mathbf{v}}$ operation, the instantaneous active component $e_{\mathrm{p}}$ and the instantaneous reactive component $e_{\mathrm{q}}$ of voltage, the instantaneous active component $i_{\mathrm{p}}$ and the instantaneous reactive component $i_{\mathrm{q}}$ of current are obtained. The DC component of the instantaneous reactive current $i_{\mathrm{qm}}$ is obtained by the current average value filter. Similarly, the DC component of the instantaneous voltage $e_{\mathrm{pm}}$ and $e_{\mathrm{qm}}$ can be obtained. The DC component of active current $i_{\text {pm }}$ is adjusted by PI controller after the STATCOM DC side capacitor voltage $U_{\mathrm{dc}}$ is subtracted from a given value $U_{\mathrm{dc} \_ \text {ref. }}$. Based on the expression (13)-(14) and the inverse matrix $\mathbf{C}_{v}^{-1}$ of the virtual matrix, the component of the compensation instruction in two phase orthogonal coordinate system $i_{\alpha f}, i_{\beta f}$ are obtained. After $\alpha-\beta /$ abc coordinate transformation, the compensation current $i_{\text {aref }}, i_{\text {bref }}$ and $i_{\text {cref }}$ are obtained. The STATCOM output current $i_{\mathrm{sa}}, i_{\mathrm{sb}}$ and $i_{\mathrm{sc}}$ are subtracted from the compensation current instruction signal respectively. The signals are fed into a sinusoidal pulse width modulator by the respective PI regulator, and the inverter bridge circuit is triggered after the triangular carrier signal modulation.

\subsection{STATCOM parameter design}

In this paper, the capacity of STATCOM $Q=1 \mathrm{Mvar}$; the DC side voltage $U_{\mathrm{dc}}=2400 \mathrm{~V}$; the parameters of transformer T: the capacity $S_{\mathrm{N}}=2 \mathrm{Mvar}$, the primary side voltage $U_{1 \mathrm{~N}}=35 \mathrm{kV}$, the secondary side voltage $U_{2 \mathrm{~N}}=690 \mathrm{~V}$.

\subsubsection{DC side capacitor selection}

The effective value of reactive current for STATCOM is:

$$
I_{e}=\frac{Q}{3 U_{2 \mathrm{~N}}}=\frac{1 \times 10^{6}}{3 \times 690}=483(\mathrm{~A})
$$

In this paper, the DC side capacitance value is calculated according to the following empirical formula:

$$
C=\frac{0.2 I_{e}}{\omega U_{\mathrm{dc}} K}=\frac{0.2 I_{e}}{2 \pi f U_{\mathrm{dc}} K}
$$

$K$ is the DC side voltage fluctuation coefficient; $f$ is the grid frequency. Select $K=0.01$, the value of the DC side capacitor estimated by the equation (16) is $12.8 \mathrm{mF}$.

\subsubsection{Connection reactor selection}

In this paper, the reactor parameter is calculated according to the following empirical formula:

$$
L \leq \frac{3 U_{2 \mathrm{~N}}\left(0.353 m_{\max } U_{\mathrm{dc}}-U_{2 \mathrm{~N}}\right)}{314 Q}
$$

$U_{2 \mathrm{~N}}$ is the effective value of the secondary side voltage; $U_{\mathrm{dc}}$ is the DC side voltage of STATCOM; $m_{\max }$ is the maximum modulation ratio. The SPWM modulation method is used in this paper, so $m_{\max }=0.9$. The reactor parameter calculated by the equation (17) is $0.4 \mathrm{mH}$. 


\subsection{Simulation modelling and analysis}

According to the STATCOM improved control strategy diagram shown in Fig. 4, the simulation model of STATCOM is built in Matlab/Simulink environment, as shown in Fig. 5.

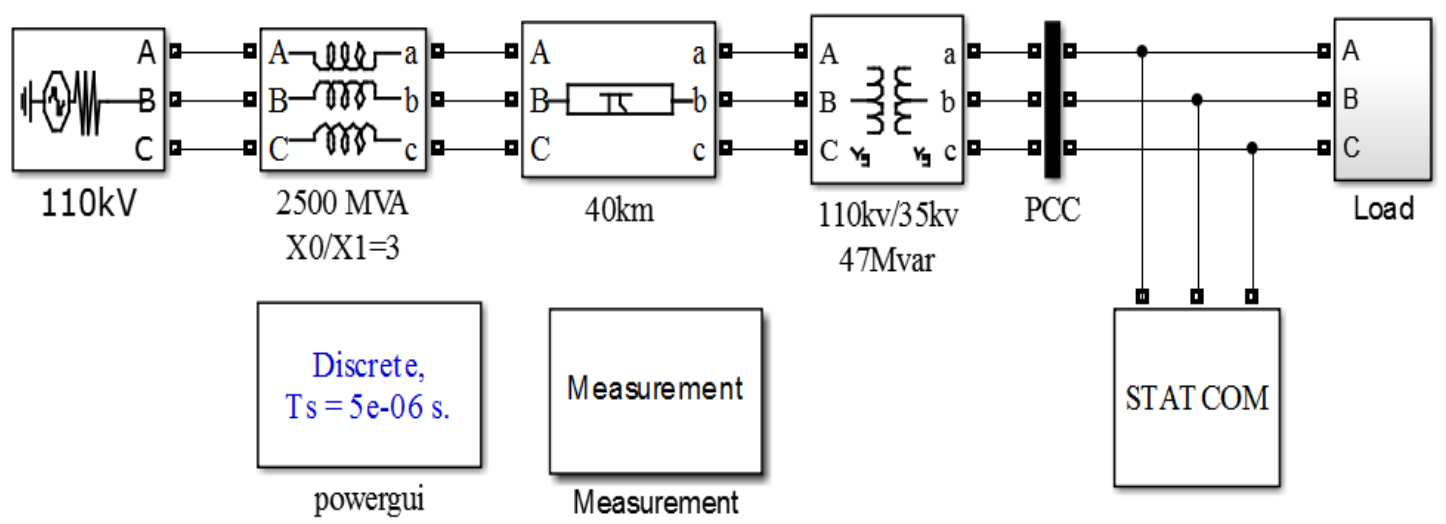

Figure 5. Simulation model of STATCOM.

\subsubsection{Inductive load}

The working conditions are as follows: the perceptual load capacity is $0.4 \mathrm{MW}$ during $0 \sim 0.2 \mathrm{~s}, 1 \mathrm{MW}$ during $0.2 \sim 0.3 \mathrm{~s}, 0.6 \mathrm{MW}$ during $0.3 \sim 0.4 \mathrm{~s}$; at $0.4 \mathrm{~s}$, STATCOM is broken away from network. Simulation results are shown in Fig. 6.

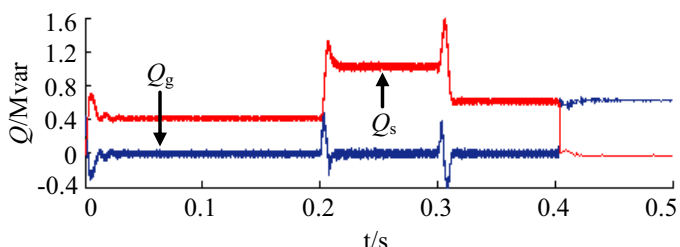

(a) Reactive power

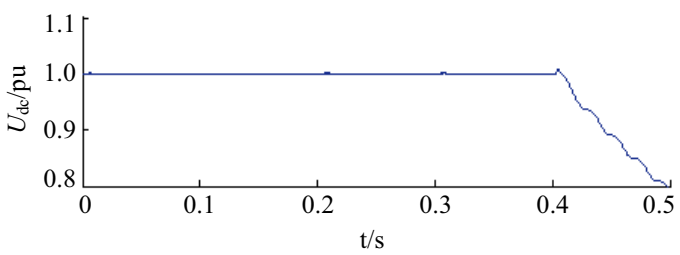

(c) DC side capacitor voltage

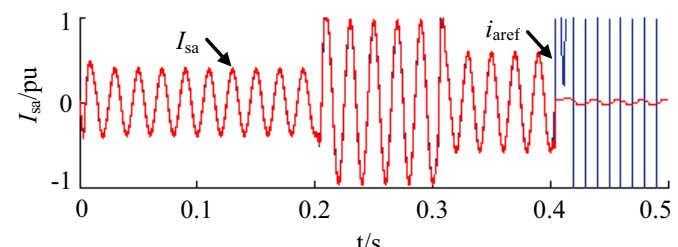

(b) STATCOM output current of A-phase

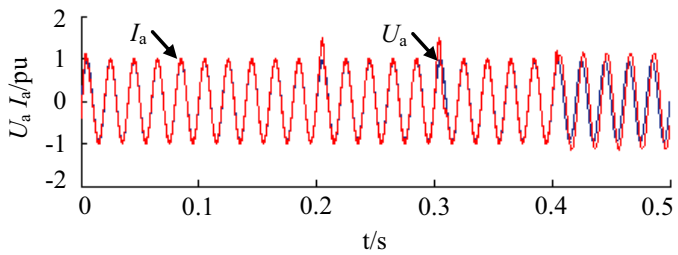

(d) Network side voltage and current of A-phase

Figure 6. Simulation waveforms of inductive load.

Fig. (a) shows the reactive power of the network side output and the STATCOM output. During $0 \sim 0.4 \mathrm{~s}$, the required reactive power of the load is supplied by STATCOM, while the reactive power of the STATCOM output is compensated with the reactive power demand. The reactive power of the load is supplied by the power grid after $0.4 \mathrm{~s}$. The reactive power compensation effect of STATCOM is verified. Fig. (b) shows the A-phase of reactive power compensation reference current $I_{\text {aref }}$ and STATCOM output current $I_{\mathrm{sa}} . I_{\mathrm{sa}}$ can track the change of $I_{\text {aref }}$ during $0 \sim 0.4 \mathrm{~s}$, and $I_{\mathrm{sa}}$ is reduced to 0 at $0.4 \mathrm{~s}$, the reactive power compensation instruction current increases with the load change. Fig. (c) shows the DC side capacitor voltage $U_{\mathrm{dc}}=1$ pu during $0 \sim 0.4 \mathrm{~s}$, the capacitor can provide reliable DC voltage support. Fig. (d) shows the A-phase of network side voltage $U_{\mathrm{a}}$ and current $I_{\mathrm{a}} . U_{\mathrm{a}}$ and $I_{\mathrm{a}}$ are in 
the same phase during $0 \sim 0.4 \mathrm{~s}$. The current $I_{\mathrm{a}}$ lags behind voltage $U_{\mathrm{a}}$ after $0.4 \mathrm{~s}$, as the SATATCOM is broken away from network.

\subsubsection{Capacitive load}

The working conditions are as follows: the capacitive load capacity is $0.4 \mathrm{MW}$ during $0 \sim 0.2 \mathrm{~s}, 1 \mathrm{MW}$ during $0.2 \sim 0.3 \mathrm{~s}, 0.6 \mathrm{MW}$ during $0.3 \sim 0.4 \mathrm{~s}$; at $0.4 \mathrm{~s}$, STATCOM is broken away from network. Simulation results are shown in Fig. 7.

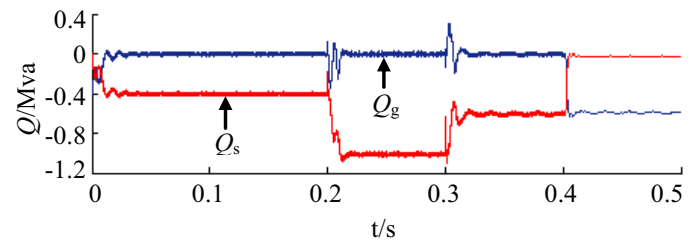

(a) Reactive power

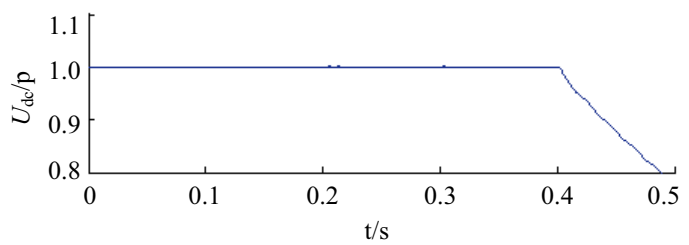

(c) DC side capacitor voltage

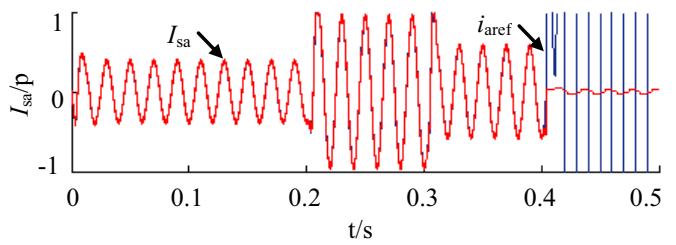

(b) STATCOM output current of A-phase

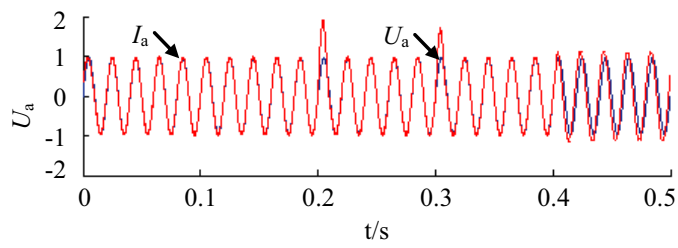

(d) Network side voltage and current of A-phase

Figure 7. Simulation waveforms of capacitive load.

Fig. (a) shows the reactive power of the network side output and the STATCOM output. During $0 \sim 0.4 \mathrm{~s}$, the required reactive power of the load is supplied by STATCOM. The reactive power of the load is supplied by the network after $0.4 \mathrm{~s}$. Fig. (b) shows the A-phase of reactive power compensation reference current $I_{\text {aref }}$ and STATCOM output current $I_{\mathrm{sa}} . I_{\mathrm{sa}}$ can track the change of $I_{\text {aref }}$ during $0 \sim 0.4 \mathrm{~s}$, and $I_{\mathrm{sa}}$ is reduced to 0 at $0.4 \mathrm{~s}$, the system no longer has the ability to compensate for reactive power. Fig. (c) shows the DC side capacitor voltage $U_{\mathrm{dc}}=1$ pu during $0 \sim 0.4 \mathrm{~s}$, the capacitor can provide reliable DC voltage support. Fig. (d) shows the A-phase of network side voltage $U_{\mathrm{a}}$ and current $I_{\mathrm{a}}$. $U_{\mathrm{a}}$ and $I_{\mathrm{a}}$ are in the same phase during $0 \sim 0.4 \mathrm{~s}$. The current $I_{\mathrm{a}}$ ahead of voltage $U_{\mathrm{a}}$ after $0.4 \mathrm{~s}$, as the SATATCOM is broken away from network.

\section{Conclusion}

Aiming at deviation and delay of the traditional reactive current detection algorithm with PLL and LPF of STATCOM, a novel improved reactive current detection algorithm without PLL is proposed, in which the VM is built to replace the original PLL, and improved current average value filter is used to realize the function of LPF. The improved control strategy for STATCOM is designed based on the VM detection method. Based on Matlab/Simulink platform, simulation analysis of the detection algorithm and control strategy is conducted so as to verify the correctness and effectiveness of the control strategy. The work done in this paper lays the good theoretical foundation for further study of STATCOM.

\section{Acknowledgement}

This work was supported by National Natural Science Foundation of China (51267015) and Specialized Research Fund for the Doctoral Program of Higher Education of China (20121514110005). 


\section{References}

1. Y. B. Guo, X. Zhou, X. H. Zhang, Electric Power Automation Equipment 32, 50 (2012)

2. C. P. Wang, X. G. Yin, Q. Xiong, Power System Protection and Control 40, 121 (2012)

3. D. Z. Li, B. Y. Chen, Electrical Measurement \& Instrumentation 48, 49 (2011)

4. X. L. Yao, L. F. Luo, J. Z. Xu, Advanced Technology of Electrical Engineering and Energy 32, $76(2013)$

5. Z. Z. Song, X. P. Ma, Y. Wang, Electrical Measurement \& Instrumentation 49, 57 (2012)

6. S. Wang, L.X Li, Y. H. Zheng, Electric Power Automation Equipment 32, 42 (2012)

7. X. R. Chang, H. Y. Wang, H. S. Zhang, Power System Technology 37, 2819 (2013)

8. G. Q. Wang, H. S. Sun, X. Y. Zhu, Automation of Electric Power Systems 37, 33 (2013)

9. X. R. Chang, H. Y. Wang, Y. J. Guo, Power System Protection and Control 42, 117 (2014)

10. G.Y. Liu, S. P. Lig, Z. Q. Qin, Power System Technology 34, 87 (2010)

11. Y. Wang, X. M. Mu, C. Qi, Power System Protection and Control 43, 131 (2015)

12. K. Chatterjee, A. Chandra, K. A. Haddad, 36th IEEE Power Electronics Specialists Conference, Brazil (2005)

13. L. J. Su, B. C. Lu, C. C. Li, Power Sysytem Protection and Control 42, 121 (2014)

14. M. K. Ghartemani, S. A. Khajehoddin, P. K. Jain, IEEE Transsactions on Power Electronics 27, $1830(2012)$

15. F. Z. Wu, S. P. Pei, 2nd International Workshop on Intelligent Systems and Applications, China (2010)

16. R. B. Gonzatti, S. C. Ferreira, D. Silva, 28th Annual IEEE Applied Power Electronics Conference and Exposition, USA (2013)

17. H. Yang, R. X. Zhao, F. B. Cheng, Proceedings of the CSEE 28, 78 (2008)

18. N. Gupta, S. P. Singh, S. P. Dubey, 5th IEEE Conference on Industrial Electronics and Applications, Taiwan (2010)

19. S. Q. Xue, J. D. Cai, Proceedings of the CSEE 32, 91 (2012)

20. L. P. Shi, P. Liu, T. R. Xu, Electrical Measurement \& Instrumentation 51, 56 (2014)

21. L. Zhou, J. Meng, Q. Liu, Power Electronics 42, 21 (2008) 